\title{
interactive effects of growth hormone and exercise on muscle mass in suspended rats
}

\author{
RICHARD E. GRINDELAND, ROLAND R. ROY, V. REGGIE EDGERTON, \\ ELENA J. GROSSMAN, VENKAT R. MUKKU, BIAN JIANG, \\ DAVID J. PIEROTTI, AND INGRID RUDOLPH \\ Life Science Division, National Aeronautics and Space Administration-Ames Research Center, \\ Moffett Field 94035; Brain Research Institute and Department of Physiological Science, \\ University of California, Los Angeles 90024-1761; and Genentech, Inc., \\ South San Francisco, California 94080
}

\begin{abstract}
Grindeland, Richard E., Roland R. Roy, V. Reggie Edgerton, Elena J. Grossman, Venkat R. Mukku, Bian Jiang, David J. Pierotti, and Ingrid Rudolph. Interactive effects of growth hormone and exercise on muscle mass in suspended rats. Am. J. Physiol. 267 (Regulatory Integrative Comp. Physiol. 36): R316-R322, 1994.-Measures to attenuate muscle atrophy in rats in response to simulated microgravity [hindlimb suspension (HS)] have been only partially successful. In the present study, hypophysectomized rats were in HS for 7 days, and the effects of recombinant human growth hormone $(\mathrm{GH})$, exercise $(\mathrm{Ex})$, or $\mathrm{GH}+\mathrm{Ex}$ on the weights, protein concentrations, and fiber cross-sectional areas (CSAs) of hindlimb muscles were determined. The weights of four extensor muscles, i.e., the soleus (Sol), medial (MG) and lateral (LG) gastrocnemius, and plantaris (Plt), and one adductor, i.e., the adductor longus (AL), were decreased by $10-22 \%$ after $\mathrm{HS}$. Fiber CSAs were decreased by $34 \%$ in the Sol and by $17 \%$ in the MG after HS. In contrast, two flexors, i.e., the tibialis anterior (TA) and extensor digitorum longus (EDL), did not atrophy. In HS rats, GH treatment alone maintained the weights of the fast extensors (MG, LG, Plt) and flexors (TA, EDL) at or above those of control rats. This effect was not observed in the slow extensor (Sol) or AL. Exercise had no significant effect on the weight of any muscle in HS rats. A combination of $\mathrm{GH}$ and $\mathrm{Ex}$ treatments yielded a significant increase in the weights of the fast extensors and in the CSA of both fast and slow fibers of the MG and significantly increased Sol weight and CSA of the slow fibers of the Sol. The AL was not responsive to either GH or Ex treatments. Protein concentrations of the Sol and MG were higher only in the Sol of $\mathrm{Ex}$ and $\mathrm{GH}+\mathrm{Ex}$ rats. These results suggest that while GH treatment or intermittent highintensity exercise alone have a minimal effect in maintaining the mass of unloaded muscle, there is a strong interactive effect of these two treatments.
\end{abstract}

countermeasures; hindlimb suspension; muscle atrophy; growth hormone; exercise; simulated microgravity

OVERLOADING OF SKELETAL MUSCLE of intact rats by surgical removal or tenotomy of synergists leads to hypertrophy of the remaining musculature, whereas unloading of muscles in hindlimb suspended (HS) or spaceflight rats and humans exposed to spaceflight results in a significant loss of muscle mass $(2,17,22,25$, $29,31,35)$. The mechanisms by which adaptations in muscle mass occur in response to these load-related changes remain unclear. It is known, however, that a host of humoral factors, including growth hormone $(\mathrm{GH})$, insulin, adrenal cortical steroids, thyroid hormones, and androgenic steroids can also affect skeletal muscle mass.
In hypophysectomized rats, $\mathrm{GH}$ treatment increases body and muscle weights $(13,14)$, stimulates muscle protein synthesis (5), and results in a positive nitrogen balance (20). Aging men and GH-deficient children also show increases in their muscle masses after treatment with $\mathrm{GH}$ (27). Hypophysectomized-HS rats show only $\sim 60 \%$ as much body and muscle growth in response to exogenous GH as ambulatory-hypophysectomized rats, indicating a degree of resistance to the hormone as a result of unloading (10). Hypertrophy of overloaded muscles and atrophy of muscles with reduced loading can occur independently of pituitary and/or pituitarydependent hormones, since these effects have been observed in hypophysectomized rats $(6,10)$. It also has been shown that aerobic or resistive exercise regimens can stimulate secretion of $\mathrm{GH}(19,23)$, whereas spaceflight and HS reduce GH secretion $(11,34)$, indicating that under physiological conditions both loading and hormonal factors can contribute to the regulation of muscle mass.

Thus the aim of the present study was to determine the interactive effects of loading by episodic exercise and $\mathrm{GH}$ on skeletal muscles of HS rats. To unequivocally distinguish loading effects from pituitary hormonal effects and their interaction on skeletal muscle, hypophysectomized rats were used. Preliminary results have been published in abstract form (12).

\section{METHODS}

Experimental animals. Male albino rats (Simonsen Laboratories, Gilroy, CA) were hypophysectomized at 49 days of age $(\sim 240 \mathrm{~g}$ body wt) and arrived at Ames Research Center 3 days posthypophysectomy. The rats were put on a reversed 12:12-h light-dark cycle and maintained at $24 \pm 1^{\circ} \mathrm{C}$ throughout the study. Animal room lights were covered with Rosco medium red filters (Rosco Laboratories, Hollywood, CA), which transmit light at wavelengths $>600 \mathrm{~nm}$ with a peak at $680 \mathrm{~nm}$. Use of the filters provided a "dark" environment for the rats but permitted manipulation of the animals. Entry to the animal room was blocked by an opaque cloth, which stopped the hall light from penetrating into the room during the entry. Food and distilled water were provided ad libitum. Adaptation to the suspension cages ( $36 \times 36 \times 42 \mathrm{~cm}$ with Plexiglas walls and a grid floor) and to the powdered food (Purina rat chow) began 2 days before the onset of the study. Animal care and use were in accord with the Ames Research Center Animal Users Guide (AHB 7180) and the Guidelines of the National Insticutes of Health and were approved by the institutional Animal Care and Use Committee.

The rats were accustomed to ladder climbing for 3 days $(7,8$, 15,16 ). The ladder was a 1 -m grid (inclined at $85^{\circ}$ ) composed of 
plastic-coated $1 / 2$-in. mesh hardware cloth. The rats were placed at the bottom of the ladder, and once they climbed to the top, they were immediately placed back at the bottom until they completed each bout of exercise (Ex). Weights (plastic film canisters filled with lead shots) were attached to the base of the tail with Velcro and were adjusted to the weight of each rat every other day during the experiment. The rats first climbed without any weight attached, and then the weight was gradually increased to $20 \%$ of body weight. The average climbing duration lasted $\sim 6-10 \mathrm{~min}$. Rats that refused to climb or showed unusual weight changes during the adaptation period were excluded from the study. During the last few days of the study, two rats in the HS + GH+Ex group did not consistently complete the required number of climbs. These rats did maintain a static position on the ladder, however, and were taken down after $\sim 30 \mathrm{~min}$. Because there were no overt differences in body or tissue weights between these two rats and the other rats in their group, they were included in the statistical analyses.

Nine days posthypophysectomy, the 7-day suspension study began. The rats (average weight $205 \pm 1 \mathrm{~g}$ ) were randomly assigned to one of six treatment groups ( $n=6 /$ group): ambulatory plus saline $(\mathrm{Amb}+\mathrm{Sal})$, Amb plus GH treatment $(\mathrm{Amb}+\mathrm{GH})$, HS plus saline (HS+Sal), HS plus GH $(\mathrm{HS}+\mathrm{GH})$, HS plus saline plus exercise treatment (HS+Sal+Ex), and $\mathrm{HS}+\mathrm{GH}+\mathrm{Ex}$. The rats were suspended using a modification of the technique initially described by Wronski and MoreyHolton (35). Briefly, the tail was cleaned with ethanol and sprayed with a benzoin-isopropyl alcohol mixture to protect the tail from irritation. The benzoin-alcohol was dried using a hair dryer. Approximately two-thirds of the tail was then wrapped in a piece of Fast-Trac adhesive tape (Zimmer Distribution, San Jose, CA), covered with a stockinette (American Hospital Supply) and secured with fiber tape. The FastTrac tape ( $R$ ) was passed through a wire hook, which was then suspended from a fishing swivel. The swivel was, in turn, suspended from the overhead track system. This arrangement allowed the rats to move freely about the cage on their forelimbs. The suspension tracks were blocked, so that the rats were unable to touch the sides of the cage with their hindlimbs.

The exercise regimen consisted of three bouts (9:00 A.M., 1:00 P.M., and 4:30 P.M.) of five climbs up a ladder while carrying a load equal to $20 \%$ of their body weight attached to the tail harness (16). The load borne by the rats during climbing was near the maximal load that these hypophysectomized rats would carry repeatedly over the course of the study. Intact HS rats have been shown to carry much heavier loads (16). The choice of multiple bouts per day was based on the finding that short multiple bouts of exercise are a better inhibitor of atrophy in HS rats than a single longer bout of exercise (15). To complete the exercise regimen, the rats were free to fully support their weight for 6-10 $\mathrm{min} /$ day. Nonexercised rats were handled 3 times/day but not allowed to exert force with their hindlimbs. All rats were weighed every other day, and the loads were adjusted to body weight. HS rats were weighed while suspended. The harness typically weighed $4-5$ g. The reported body weights are corrected for the weight of the harness.

The rats in the $\mathrm{Amb}+\mathrm{GH}, \mathrm{HS}+\mathrm{GH}$, and $\mathrm{HS}+\mathrm{GH}+\mathrm{Ex}$ groups were injected subcutaneously with recombinant human GH (rhGH, Genentech, South San Francisco, CA) at a dosage of $1 \mathrm{mg} \cdot \mathrm{kg}^{-1} \cdot$ day $^{-1}$. Rats in the corresponding control groups were injected with a similar volume $(0.5 \mathrm{ml})$ of saline. Injections were given $\sim 30$ min before each exercise session.

The rats were killed by decapitation. Completeness of hypophysectomy was verified by inspection of the sella turcica and by determination of adrenal and testis weights. The adrenal glands and the testis were removed bilaterally, cleaned of fat and connective tissue, and weighed to the nearest $0.1 \mathrm{mg}$. The tibia on one side was prepared for tibial plate analysis (9). Four muscles, the soleus (Sol), medial gastrocnemius (MG), tibialis anterior (TA), and adductor longus (AL) were removed bilaterally and weighed (wet weight). Three muscles, the lateral gastrocnemius (LG), plantaris (Plt), and extensor digitorum longus (EDL), were removed unilaterally and weighed. The Sol and MG from one side were quick-frozen in liquid nitrogen and stored at $-70^{\circ} \mathrm{C}$ until used for protein determinations. The Sol and MG on the contralateral side were frozen in Freon- 12 cooled by liquid nitrogen and then stored at $-70^{\circ} \mathrm{C}$ until used for histological and histochemical analyses. A zero time $\left(\mathrm{T}_{0}\right)$ control group of six rats, averaging $210 \mathrm{~g}$ body wt, was killed on the day suspension began. In this group, the testis and three muscles, Sol, TA, and whole gastrocnemius (Gast), were removed, trimmed of extraneous tissue, and weighed.

Muscle histological procedures. Cross sections $(10 \mu \mathrm{m}$ thick) were cut in a cryostat maintained at a temperature of $-20^{\circ} \mathrm{C}$. The sections were stained for myofibrillar adenosinetriphosphatase (ATPase) at an alkaline $\mathrm{pH}$ using the methods described by Nwoye and co-workers (24). The cross-sectional areas (CSAs) of a population of 100-200 fibers from each Sol and MG muscle were determined, and the fibers were classified as dark or light staining for the myofibrillar ATPase stain.

Muscle protein determinations. For protein determinations in the Sol and MG, a piece of the midregion of each muscle was removed and homogenized in glass-distilled water $(2.5 \mathrm{mg}$ tissue $/ \mathrm{ml}$ ) using an all-glass hand homogenizer. Noncollagenous protein was determined using the BCA Protein Assay Reagent (Pierce Chemical, Rockford, IL) and recrystallized bovine serum albumin (Sigma Chemical, St. Louis, MO) as the standard (28).

Tibial growth plate measurements. Tibial epiphyseal widths were measured using the procedure of Greenspan and coworkers (9). Briefly, one tibia was dissected from each rat, split longitudinally, and stained with $\mathrm{AgNO}_{3}$. Ten readings were taken across the proximal growth plate (silver line), using an ocular micrometer. The 10 readings were averaged for each animal.

Statistics. A one-way analysis of variance was used to test for significant differences between groups, and the null hypothesis was rejected at $P<0.05$.

\section{RESULTS}

At the time of hypophysectomy, the rats weighed $\sim 240 \mathrm{~g}$ and 9 days later, at the beginning of the suspension period, weighed an average of $205 \mathrm{~g}$. The posthypophysectomy decrease $(15 \%)$ in body weight is typical, in our experience, for rats of this size (R. E. Grindeland and E. J. Grossman, unpublished observations). During the 7-day experimental period, the rats in the Amb+Sal, HS+Sal, and HS+Sal+Ex groups lost an additional 9 or $10 \mathrm{~g}$ (Table 1). In contrast, Amb+GH rats gained $39 \mathrm{~g}$, whereas $\mathrm{HS}+\mathrm{GH}$ and $\mathrm{HS}+\mathrm{GH}+\mathrm{Ex}$ rats gained 23 and $22 \mathrm{~g}$, respectively, $\sim 60 \%$ as much as their $\mathrm{Amb}+\mathrm{GH}$ controls. Weight gains of $\mathrm{HS}+\mathrm{GH}$ and $\mathrm{HS}+\mathrm{GH}+\mathrm{Ex}$ rats were significantly less $(P<0.01)$ than those of $\mathrm{Amb}+\mathrm{GH}$ animals. There was no effect of exercise nor any interaction of $\mathrm{GH}$ and exercise on body weights of HS rats.

Adrenal weights of the Amb + Sal rats were $\sim 50 \%$ of those found for intact rats of similar body weight (R. E. 
Table 1. Body and adrenal weights and tibial epiphyseal widths of hypophysectomized rats hindlimb suspended and treated with growth hormone, exercise, or growth hormone and exercise

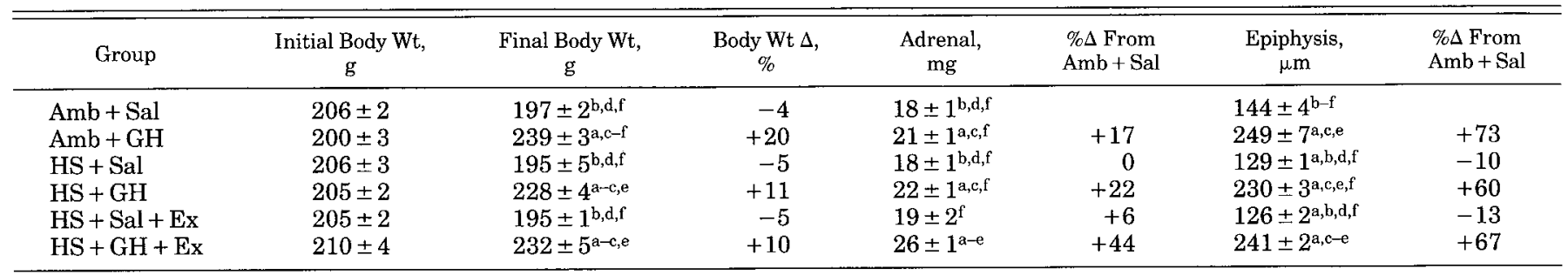

Values are means \pm SE. $\Delta$, change; Amb, ambulatory; Sal, saline; HS, hindlimb suspended; GH, growth hormone; Ex, exercise; for further description of groups see METHODS. Differs $(P<0.05)$ from Amb + Sal ${ }^{(a)}, \mathrm{Amb}+\mathrm{GH}\left({ }^{\mathrm{b}}\right), \mathrm{HS}+\mathrm{Sal}\left({ }^{\mathrm{c}}\right), \mathrm{HS}+\mathrm{GH}\left({ }^{\mathrm{d}}\right), \mathrm{HS}+\mathrm{Sal}+\mathrm{Ex}\left({ }^{(e)}\right)$ or HS + $\mathrm{GH}+\operatorname{Ex}\left({ }^{f}\right)$.

Grindeland and E. J. Grossman, unpublished observations), verifying that the rats were completely hypophysectomized. GH treatment resulted in similar increments in adrenal weights in both Amb and HS rats. Exercise alone had no effect on adrenal weights. However, the adrenal weight response was greater in the $\mathrm{HS}+\mathrm{GH}+\mathrm{Ex}$ than the $\mathrm{Amb}+\mathrm{GH}$ or $\mathrm{HS}+\mathrm{GH}$ groups, indicating a $\mathrm{GH}+\mathrm{Ex}$ interaction. Whether the adrenal hypertrophy was due to growth of cortical or medullary tissue, or both, was not investigated. Testis weights $(1,356 \pm 42 \mathrm{mg})$ of $\mathrm{Amb}+$ Sal rats were reduced $\sim 70 \%$ from those of intact rats of similar body weight (R. E. Grindeland, unpublished observations), further supporting the completeness of the hypophysectomy. Testis weights of suspended (HS+Sal) rats were $31 \%$ smaller than Amb +Sal rats $(P<0.05)$.

As expected, the tibial growth plates showed marked growth in $\mathrm{Amb}+\mathrm{GH}$ rats (Table 1). Suspended rats given GH also showed significant growth in epiphyseal plate thickness, and the differences in epiphyseal widths between the appropriate control group and the $\mathrm{Amb}+\mathrm{GH}$ or $\mathrm{HS}+\mathrm{GH}$ groups were similar. Interestingly, the $\mathrm{HS}+$ Sal rats exhibited a small but significant $(15 \mu \mathrm{m})$ decrement in growth plate widths compared with $\mathrm{Amb}+$ Sal, suggesting an inhibition of autonomous bone growth in the HS rats. Exercise (HS+Sal+Ex) by itself did not counter the inhibition of tibial plate growth seen in HS+Sal rats. However, exercise did interact with GH in $\mathrm{HS}+\mathrm{GH}+\mathrm{Ex}$ rats to yield a small but significant increase in growth plate thickness compared with $\mathrm{HS}+\mathrm{GH}$ rats, resulting in values that were not different from those of $\mathrm{Amb}+\mathrm{GH}$ rats.

Comparison of muscle weights of rats killed at the beginning of suspension ( $\mathrm{T}_{0}$ control) and $\mathrm{Amb}+\mathrm{Sal}$ rats killed at the end of the experiment showed no effect of hypophysectomy on the weight of the Sol but atrophy of 11 and 28\%, respectively, for the Gast and TA (Table 2).

Table 2. Rat muscle weights

\begin{tabular}{lcccl}
\hline \hline Treatment & $\begin{array}{c}\text { Days After } \\
\text { Surgery }\end{array}$ & Sol & Gast & TA \\
\hline $\mathrm{T}_{0}$ & 9 & $97 \pm 4^{\mathrm{c}}$ & $1,309 \pm 27^{\mathrm{b}, \mathrm{c}}$ & $553 \pm 29^{\mathrm{b}, \mathrm{c}}$ \\
$\mathrm{Amb}+$ Sal & 16 & $98 \pm 4^{\mathrm{c}}$ & $1,164 \pm 5^{\mathrm{a}, \mathrm{c}}$ & $398 \pm 6^{\mathrm{a}}$ \\
$\mathrm{HS}+\mathrm{Sal}$ & 16 & $81 \pm 3^{\mathrm{a}, \mathrm{b}}$ & $1,016 \pm 21^{\mathrm{a}, \mathrm{b}}$ & $385 \pm 10^{\mathrm{a}}$ \\
\hline
\end{tabular}

Values are means \pm SE. Muscle weights in mg. Sol, Soleus; Gast, gastrocnemius; TA, tibialis anterior. Differs $(P<0.05)$ from 0 time group $\left(\mathrm{T}_{0}\right){ }^{(\mathrm{a})}, \mathrm{Amb}+\mathrm{Sal}\left({ }^{\mathrm{b}}\right), \mathrm{HS}+\mathrm{Sal}\left({ }^{(}\right)$.
Hindlimb suspension (HS+Sal) resulted in atrophy of the Sol and further atrophy of the Gast, but had no effect on the TA.

GH treatment had little effect on the mass of either the Sol or AL, i.e., two muscles comprised predominantly of slow fibers, of Amb rats (Fig. 1). As expected, HS resulted in a decrease in Sol and AL mass of 17 and $22 \%$ compared with Amb+Sal. GH or exercise treatments alone were ineffective in countering this atrophic response in either muscle. However, the combined effects of GH and exercise in the suspended rat restored the Sol weight to those of $\mathrm{Amb}+\mathrm{Sal}$ and $\mathrm{Amb}+\mathrm{GH}$ rats, demonstrating a clear interaction of $\mathrm{GH}$ and exercise on this muscle. In contrast, there was no interactive effect on the AL of HS rats.

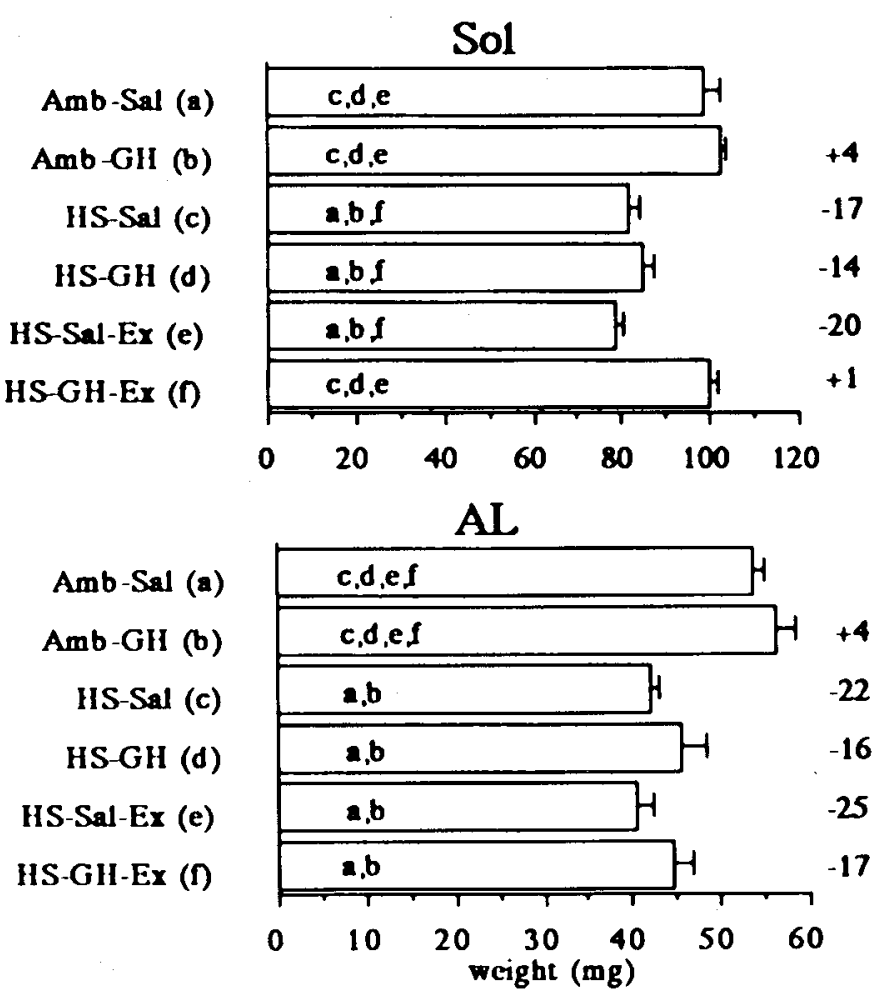

Fig. 1. Mean wet weights (SE) of soleus (Sol) and adductor longus (AL) muscles for each experimental group. Letters within each bar indicate which groups were significantly different $(P<0.05)$. Nos. to right of each bar indicate \%difference from ambulatory + saline (Amb+Sal) group. HS, hindlimb suspended; $\mathrm{GH}$, growth hormone; $\mathrm{Ex}$, exercise (see METHODs for further group description). 
Three predominantly fast extensor muscles, the MG, LG, and Plt, were affected similarly by the treatments (Fig. 2). Compared with Amb+Sal, all three muscles were significantly smaller after suspension (HS+Sal) and significantly larger after $\mathrm{GH}$ treatment $(\mathrm{Amb}+\mathrm{GH})$. Ladder climbing alone had no effect on muscle weights, with the HS+Ex rats having values similar to those of $\mathrm{HS}+$ Sal rats. In contrast, GH treatment, with or without exercise, of suspended rats restored the weights of all three muscles to Amb + Sal levels $(P>0.05)$; only the Plt of $\mathrm{HS}+\mathrm{GH}+\mathrm{Ex}$ rats was similar in weight to $\mathrm{Amb}+\mathrm{GH}$ rats. Only the $\mathrm{MG}$ weight was larger in the $\mathrm{HS}+\mathrm{GH}+\mathrm{Ex}$ than in the HS+GH group, indicating an interactive effect of $\mathrm{GH}$ and Ex.

The two predominantly fast flexors, the TA and EDL, showed significant increases in weight in response to GH treatment in both Amb and HS groups (Fig. 3). In

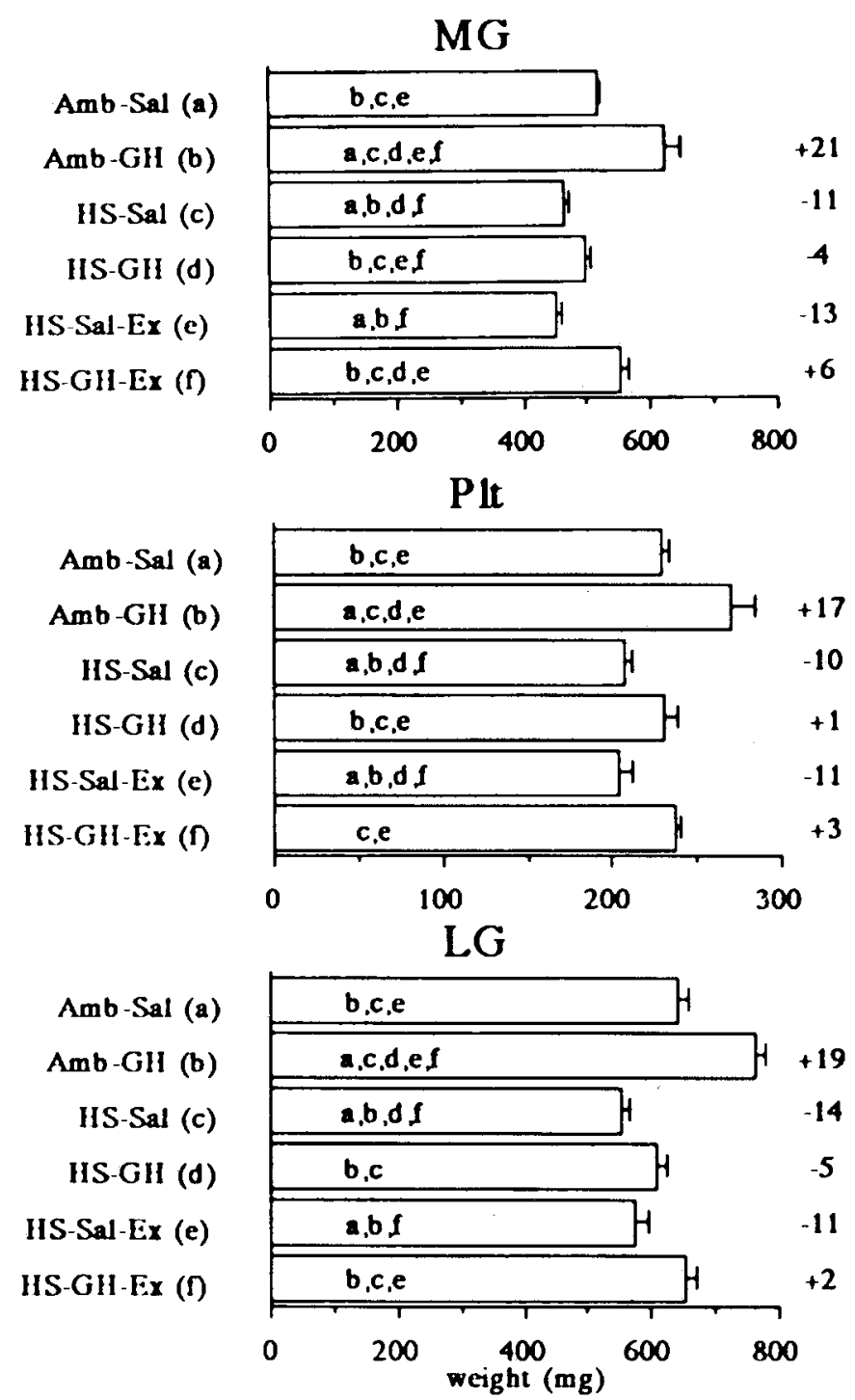

Fig. 2. Mean wet weights (SE) of medial gastrocnemius (MG), plantaris (Plt), and lateral gastrocnemius (LG) muscles for each experimental group. Letters within each bar indicate which groups were significantly different $(P<0.05)$. Nos, to right of each bar indicate \%difference from Amb + Sal.

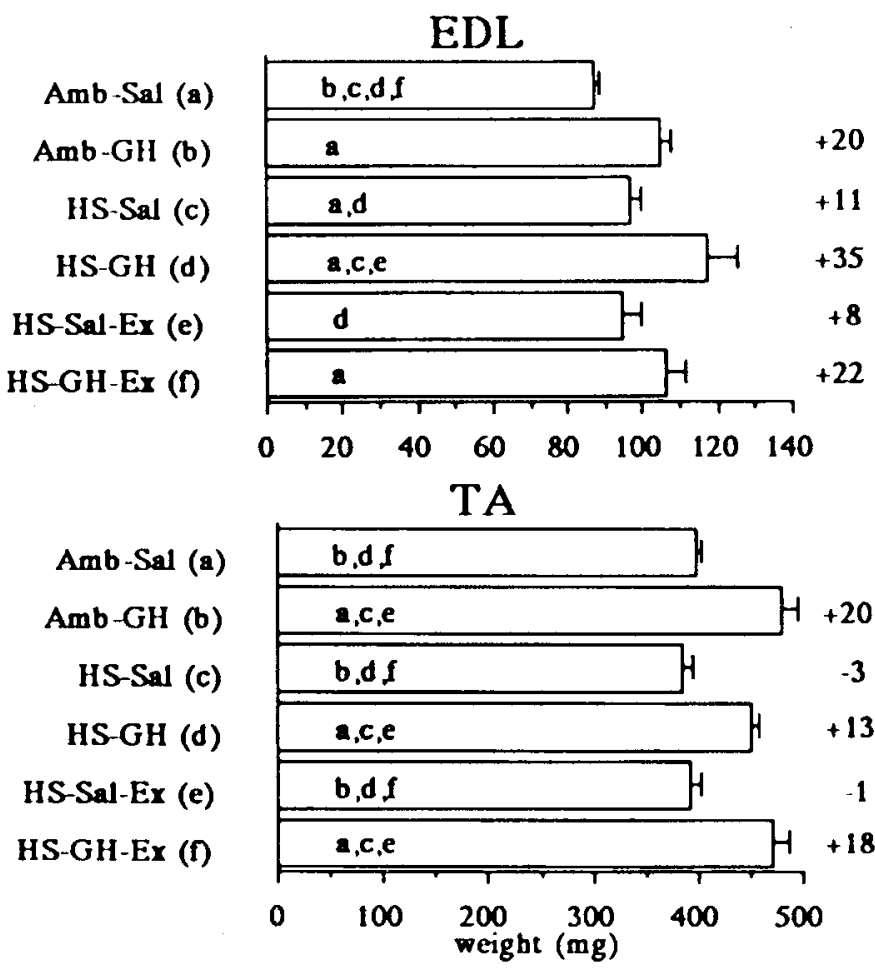

Fig. 3. Mean wet weights (SE) of extensor digitorum longus (EDL) and tibialis anterior (TA) muscles for each experimental group. Letters within each bar indicate which groups were significantly different $(P<0.05)$. Nos. to right of each bar indicate \%difference from Amb + Sal.

contrast to the predominantly slow and the predominantly fast extensor muscles, the flexors did not atrophy during HS. In fact, the EDL was significantly larger in $\mathrm{HS}+\mathrm{Sal}$ than in Amb+Sal rats. The exercise regimen had no detectable effect on the mass of either flexor muscle in HS rats. A combination of GH and exercise treatments, however, resulted in TA masses that were significantly larger than those observed in Amb+Sal, $\mathrm{HS}+\mathrm{Sal}$, and $\mathrm{HS}+\mathrm{Sal}+\mathrm{Ex}$ groups and similar to those in $\mathrm{HS}+\mathrm{GH}$ and $\mathrm{Amb}+\mathrm{GH}$ rats. Together, these data indicate that the growth of the TA in HS rats was attributable to $\mathrm{GH}$ treatment alone. For the EDL, $\mathrm{HS}+\mathrm{GH}+\mathrm{Ex}$ rats had heavier muscles than Amb+Sal animals, but they were not significantly different from those of any other group.

Noncollagenous protein concentrations of the Sol and MG varied from 14.5 to $16.6 \%$ of wet weight for all treatment groups (Table 3 ). The only significant differ-

Table 3. Muscle protein concentrations

\begin{tabular}{lrr}
\hline \hline \multicolumn{1}{c}{ Group } & Sol & \multicolumn{1}{c}{ MG } \\
\hline Amb + Sal & $14.6 \pm 0.2$ & $15.2 \pm 0.5$ \\
Amb + GH & $15.3 \pm 0.5$ & $15.5 \pm 0.4$ \\
HS + Sal & $15.9 \pm 0.7$ & $14.5 \pm 0.6$ \\
HS + GH & $15.4 \pm 0.8$ & $14.5 \pm 0.3$ \\
HS + Sal + Ex & $16.6 \pm 0.5^{\mathrm{a}}$ & $15.9 \pm 0.7$ \\
HS + GH + Ex & $15.9 \pm 0.5^{\mathrm{a}}$ & $16.2 \pm 0.8$ \\
\hline
\end{tabular}

Values are means \pm SE. Muscle protein concentration given as $\%$ wet wt. MG, medial gastrocnemius. Differs $(P<0.05)$ from Amb + Sal (a). 


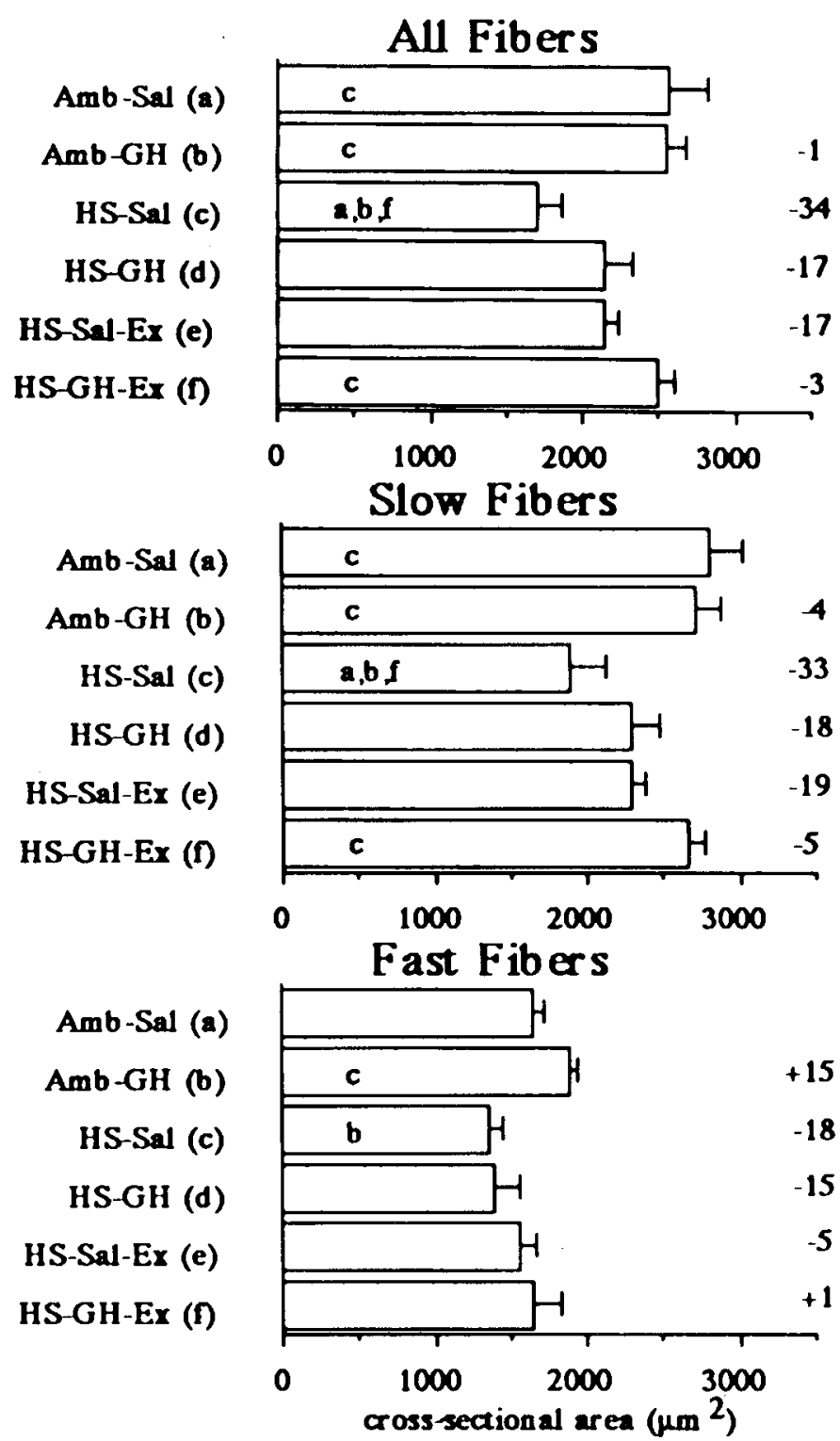

Fig. 4. Mean cross-sectional areas (SE) for all, light ATPase (slow), and dark ATPase (fast) fibers of soleus muscles for each experimental group. Letters within each bar indicate which groups were significantly different $(P<0.05)$. Nos. to right of each bar indicate \%difference from Amb+Sal.

ences found between groups were the higher concentrations in the Sol of $\mathrm{HS}+\mathrm{Sal}+\mathrm{Ex}$ and $\mathrm{HS}+\mathrm{GH}+\mathrm{Ex}$ rats compared with Amb+Sal controls. Thus the changes in muscle weights and fiber CSAs in these two muscles cannot be attributed only to varying water content.

The pattern of changes in the CSA of the Sol and MG fibers was similar to, but not identical with, that of muscle wet weights (Figs. 4 and 5). GH had little effect on the size of the slow fibers, the predominant fiber type, in the Sol of ambulatory rats (Fig. 4). Suspension resulted in a significant decrease in the mean CSA of the slow fibers in the Sol compared with either the Amb+Sal or Amb+GH rats (Fig. 4). Either exercise or GH alone ameliorated (by $\sim 45 \%$ compared with $\mathrm{Amb}+\mathrm{Sal}, P>$ 0.05 ) but did not prevent the suspension-induced atrophic response, whereas there was no evidence of a GH or Ex effect on the Sol based on wet muscle weight. The slow fibers in the HS+GH+Ex group were only $\sim 5 \%$ smaller $(P>0.05)$ than the Amb+Sal and virtually identical to the $\mathrm{Amb}+\mathrm{GH}$ fibers. In addition, the slow fibers in the HS+GH+Ex group were significantly larger than in the $\mathrm{HS}+\mathrm{Sal}$ rats. The mean crosssectional area of the fast fibers in the Sol of $\mathrm{Amb}+\mathrm{GH}$ rats was $\sim 15 \%$ larger $(P>0.05)$ than in the $\mathrm{HS}+\mathrm{Sal}$ rats. The only significant change, however, was that the fast fibers in the $\mathrm{HS}$ rats were smaller than in $\mathrm{Amb}+\mathrm{GH}$ rats. When all fibers (slow plus fast) were considered, the adaptations were similar to those observed for the slow fibers.

Treatment with GH resulted in no change in CSAs of either fiber type in the MG of Amb+GH rats (Fig. 5). Suspension resulted in $\sim 27 \%$ atrophy in both the fast and slow fibers compared with Amb+Sal rats. Either GH or Ex treatment alone appeared to lessen the atrophic response by $5-18 \%(P>0.05)$ compared with $\mathrm{HS}+\mathrm{Sal}$ rats, whereas the combination of these treatments maintained the fiber sizes at the level of the

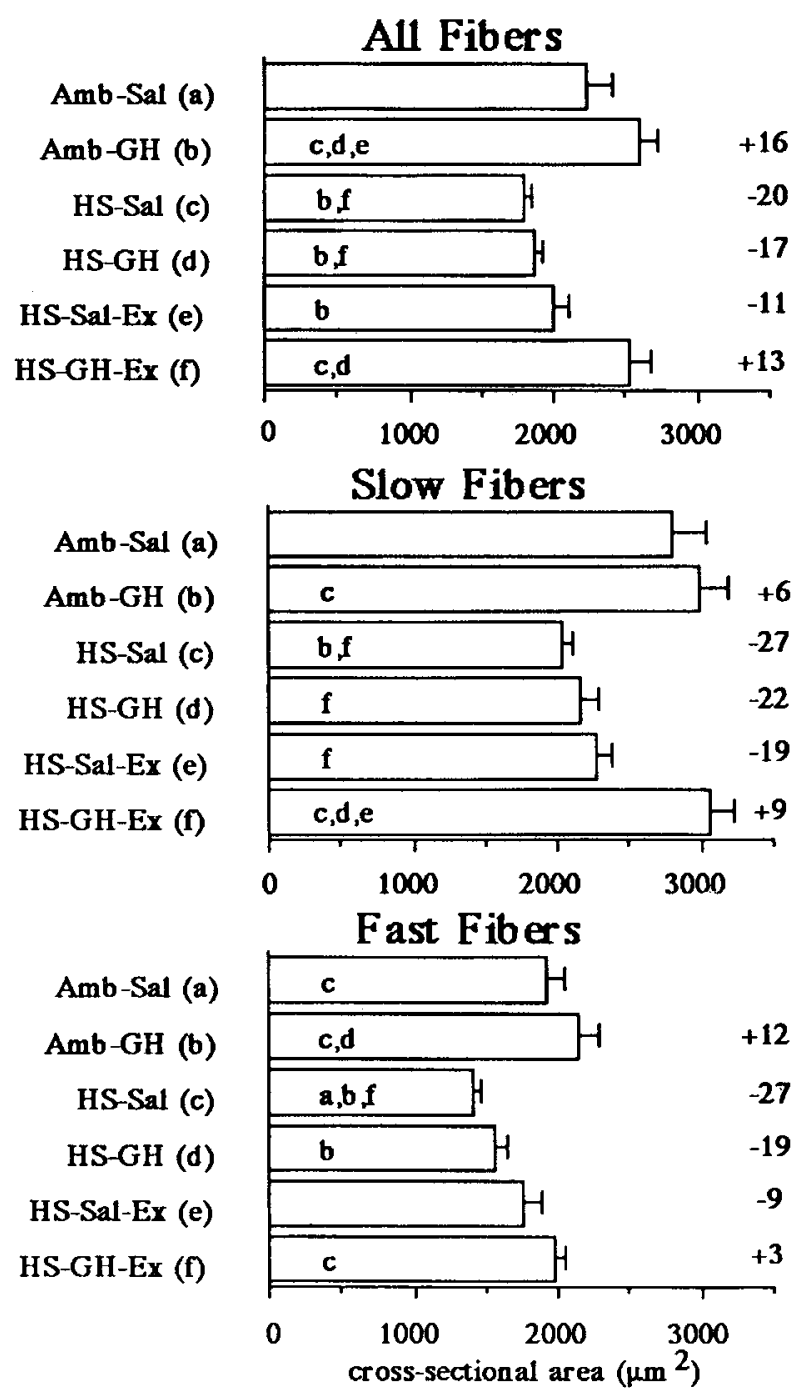

Fig. 5. Mean cross-sectional areas (SE) for all, light ATPase (slow), and dark ATPase (fast) fibers of medial gastrocnemius muscles for each experimental group. Letters within each bar indicate which groups were significantly different $(P<0.05)$. Nos. to right of each bar indicate \%difference from $\mathrm{Amb}+\mathrm{Sal}$. 
$A m b+$ Sal rats. Fiber CSAs did not differ $(P>0.05)$ between $\mathrm{HS}+\mathrm{GH}+\mathrm{Ex}$ and $\mathrm{Amb}+\mathrm{Sal}$ or $\mathrm{Amb}+\mathrm{GH}$ groups, but for the slow and for the combination of slow and fast fibers the mean CSAs were significantly larger in $\mathrm{HS}+\mathrm{GH}+\mathrm{Ex}$ than in $\mathrm{HS}+\mathrm{Sal}$ rats.

\section{DISCUSSION}

GH administration and exercise training can have an anabolic effect on skeletal muscle $(2,3,12-14,18,25$, 26,30 ), but the magnitude or even the presence of this effect depends on a number of factors, including the type of muscle, nature of the exercise training, and hormonal status of the animal. In addition, when combinations of these experimental variables are imposed simultaneously, the effects will often reflect some additive response. For example, when HS rats were treated with the anabolic steroid nandrolone decanoate, the Sol and Plt were 20-30\% larger than in HS rats not treated with the anabolic drug $(32,33)$. Furthermore, when the Sol and Plt of HS rats were overloaded by surgically removing their synergists and given the anabolic hormone, the muscle weights were $110 \%$ larger than control. In contrast, HS-overloaded rats not treated with the hormone showed only a $60 \%$ increase in muscle weight. These results suggest that the steroid increased muscle mass by $20-30 \%$, the overload increased the mass by $\sim 60 \%$, and the combined effect was $110 \%$. Thus a modest interaction of these two treatments was suggested in that the combined effect exceeded the sum of the individual effects.

In the present study, both additive ( $\mathrm{GH}$ effect $+\mathrm{Ex}$ effect $=\mathrm{GH}+\mathrm{Ex}$ effect $)$ and interactive (GH effect $+\mathrm{Ex}$ effect $<\mathrm{GH}+$ Ex effect) effects of exercise training and GH on HS rats were observed. In some instances, the additive effect can be attributed to the $\mathrm{GH}$ effect. Based on muscle wet weights, the EDL and Plt had a significant response to GH treatment, no effect of exercise treatment, and no interactive effect between the two treatments. No exercise, $\mathrm{GH}$, or interactive effect was observed in the AL. The weights of the MG, LG, and TA were increased by $\mathrm{GH}$ alone and further increased by the $\mathrm{GH}+\mathrm{Ex}$ regimen and showed a clear interactive effect between the treatments. The Sol weight was unaffected by GH alone but, like the MG, LG and TA clearly showed an interactive effect of $\mathrm{GH}$ plus exercise. Based on the CSA of fibers measured in the Sol and MG there was an additive effect of $\mathrm{GH}$ and $\mathrm{Ex}$ in the Sol and a clear interactive effect in the MG (Fig. 5). In the slow fibers of the MG, the CSA of the HS+GH rats was $135 \mu^{2}$ larger than in $\mathrm{HS}+\mathrm{Sal}$ rats. The exercise training effect was $230 \mu \mathrm{m}^{2}$. The mean fiber CSA of the HS rats that were trained and received GH was $1,027 \mu \mathrm{m}^{2}$, i.e., $181 \%$ larger than the sum of the individual treatment effects. Increments in MG fast fiber CSA were $155 \mu \mathrm{m}^{2}$ in $\mathrm{HS}+\mathrm{GH}$ rats, $348 \mu \mathrm{m}^{2}$ in HS+Ex rats, and $579 \mu \mathrm{m}^{2}$ in $\mathrm{HS}+\mathrm{GH}+\mathrm{Ex}$ animals compared with HS+Sal rats, revealing a $15 \%$ interactive effect.

The differences in responses among the muscles seem to be related to muscle function and perhaps type. In ambulatory rats, GH increased muscle mass in all fast but neither of the slow muscles. This preferential effect suggests a lesser dependence of slow than fast muscles on GH. In this study, as has been shown in others, relative muscle effects of $\mathrm{HS}$ were: slow extensors $>$ fast extensors $>$ fast flexors, suggesting a greater dependence on activity-loading of slow than fast muscle. It should be noted that in HS rats the activity (based on chronic electromyogram recordings) of the TA is markedly elevated (1) and, therefore, an activity-related decrement need not be expected. Based on muscle weight, there was no evidence of an exercise effect on any muscle, although CSA measures suggested some modest, but statistically insignificant, effect in both the Sol and MG.

Because the recruitment patterns in the Sol, MG, and TA during suspension and during the climbing exercise can be predicted (25), implications of the present study are that a combination of neuromuscular activity loading and hormonal factors can affect the muscle. While either stimulus alone may be insufficient to enhance muscle fiber size of HS rats, in some cases their combined effect can be greater than the sum of their individual effects. For example, even though the Sol of $\mathrm{HS}$ rats was only moderately responsive to $\mathrm{GH}(\mathrm{HS}+\mathrm{GH})$ or Ex (HS+Ex) treatment alone, recruitment of the muscle fibers during the climbing exercise for only a few minutes per day of GH-treated rats (HS+GH+Ex) resulted in a maintenance of mass at control levels.

As consistent as these results appear, the AL did not fit the conceptual model. Based on the response of the Sol, we would have predicted an interactive effect in the AL, also a predominantly slow muscle. Neither GH nor exercise treatment affected mass of the $\mathrm{AL}$ in HS rats. Furthermore, there was no effect when GH and exercise were combined. GH administration in the Amb or HS rats seemed to have little effect on either the Sol or $\mathrm{AL}$ wet weight. Previous experiments in our laboratory have shown that the AL can be activated during climbing (V. R. Edgerton and R. R. Roy, unpublished observations). It is possible that the AL was not sufficiently activated during the climbing task to enable a GH effect. Further work will be necessary to understand this apparently paradoxical finding in the $\mathrm{AL}$ with respect to the interactive effect of $\mathrm{GH}$ and exercise on muscle mass. The absence of an interactive effect on the Plt could reflect a minimal recruitment of this muscle during the climbing exercise, since a robust $\mathrm{GH}$ effect on the Plt was evident in the GH-treated rats.

Other than for the $\mathrm{AL}$, the $\mathrm{GH}+\mathrm{Ex}$ treatment was clearly effective in maintaining the mass of the hindlimb muscles of hypophysectomized suspended rats. Only very large doses of GH have been found to stimulate growth in ambulatory intact rats, which raises the question of whether the $\mathrm{GH}+\mathrm{Ex}$ regimen would be efficacious in the HS intact rat $(13,14)$. In a recent study, Linderman and co-workers (21) suspended intact 250 -g rats for 5 days. The rats were given saline or rhGH, exercised (ladder climbing 10 times in 3 bouts/ day while carrying a load equal to $50 \%$ of their body weight), or given $\mathrm{GH}+\mathrm{Ex}$. The combined $\mathrm{GH}+\mathrm{Ex}$ treatment maintained Gast wet weight and protein content at control levels and stimulated myofibrillar protein synthesis, while neither the GH nor the Ex treatment alone had a significant effect. 
These results suggest that skeletal muscle could be in double jeopardy during spaceflight. Intact spaceflight and HS rats have markedly reduced levels of $\mathrm{GH}$ as measured by bioassay $(10,34)$. A combination of reduced loading from either little recruitment, or little loading in spite of recruitment, and reduced circulating GH levels may make the skeletal muscle particularly susceptible to atrophy during spaceflight.

The authors thank Beth Goldman for her technical assistance and Kristin Gosselink for typing the manuscript.

This study was supported by National Aeronautics and Space Administration Grant 199-26-12-09.

Received 15 October 1993; accepted in final form 11 January 1994.

\section{REFERENCES}

1. Alford, E. K., R. R. Roy, J. A. Hodgson, and V. R. Edgerton. Electromyography of rat soleus, medial gastrocnemius, and tibialis anterior during hindlimb suspension. Exp. Neurol. 96: 635$649,1987$.

2. Edgerton, V. R., and R. R. Roy. Adaptations of skeletal muscle to spaceflight. In: Fundamentals of Space Life Sciences, edited by S. Churchill. Melbourne, Australia: Orbit Book, Krieger. In press.

3. Edgerton, V. R., and R. R. Roy. Neuromuscular responses to spaceflight. In: Handbook of Physiology. Bethesda, MD: Am. Physiol. Soc. In press.

4. Edgerton, V. R., and R. R. Roy. Neuromuscular adaptations to actual and simulated weightlessness. In: Advances in Space Biology and Medicine, edited by S. L. Bonting. Greenwich, CT: Jai In press.

5. Flaim, K. E., J. B. Li, and L. S. Jefferson. Protein turnover in rat skeletal muscle: effects of hypophysectomy and growth hormone. Am. J. Physiol. 234 (Endocrinol. Metab. Gastrointest. Physiol. 3): E38-E43, 1978.

6. Goldberg, A. L. Work-induced growth of skeletal muscles in normal and hypophysectomized rats. Am. J. Physiol. 213: 1193$1198,1967$.

7. Graham, S. C., R. R. Roy, S. P. West, D. Thomason, and K. M. Baldwin. Exercise effects on the size and metabolic properties of soleus fibers in hindlimb-suspended rats. Aviat. Space Environ. Med. 60: 226-234, 1989.

8. Graham, S. C., R. R. Roy, E. O. Hauschka, and V. R. Edgerton. Effects of weight support on medial gastrocnemius fibers of suspended rats. J. Appl. Physiol. 67: 945-953, 1989.

9. Greenspan, F. S., C. H. Li, M. E. Simpson, and H. M. Evans. Bioassay of hypophyseal growth hormone: tibia test. Endocrinology 45: 455-463, 1949.

10. Grindeland, R. E., T. N. Fast, M. Vasques, T. Satyanarayana, and M. Ruder. Does altered growth hormone physiology play a role in muscle atrophy of simulated weightlessness? NASA Life Sci. Symp. Abstr. Washington DC 1987, p. 82-83.

11. Grindeland, R. E., W. C. Hymer, M. Farrington, T. Fast, C. Hayes, K. Motter, L. Patil, and M. Vasques. Changes in pituitary growth hormone cells prepared from rats flown on Spacelab 3. Am. J. Physiol. 252 (Regulatory Integrative Comp. Physiol. 21): R209-R215, 1987.

12. Grindeland, R. E., R. R. Roy, V. R. Edgerton, E. Grossman, I. Rudolph, D. Pierotti, and B. Goldman. Exercise and growth hormone $(\mathrm{GH})$ have synergistic effects on skeletal muscle and tibias of suspended rats (Abstract). FASEB J. 5: A1071, 1991.

13. Groesbeck, M. D., A. F. Parlow, and W. H. Daughaday. Stimulation of supranormal growth in prepubertal, adult plateaued, and hypophysectomized female rats by large doses of rat growth hormone: physiological effects and adverse consequences. Endocrinology 120: 1963-1975, 1987.

14. Groesbeck, M. D., and A. F. Parlow. Highly improved precision of the hypophysectomized female rat body weight gain bioassay for growth hormone by increased frequency of injections, avoidance of antibody formation, and other simple modifications. Endocrinology 120: 2582-2590, 1987.

15. Hauschka, E. O., R. R. Roy, and V. R. Edgerton. Periodic weight support effects on rat soleus fibers after hindlimb suspension. J. Appl. Physiol. 65: 1231-1237, 1988.
16. Herbert, M. E., R. R. Roy, and V. R. Edgerton. Influence of one-week hindlimb suspension and intermittent high load exercise on rat muscles. Exp. Neurol. 102: 190-198, 1988.

17. Jiang, B., Y. Ohira, R. R. Roy, Q. Nguyen, E. I. IlyinaKakueva, V. Oganov, and V. R. Edgerton. Adaptation of fibers in fast-twitch muscles of rats to spaceflight and hindlimb suspension. J. Appl. Physiol. 73, Suppl.: 58S-65S, 1992.

18. Kirby, C. R., M. J. Ryan, and F. W. Booth. Eccentric exercise training as a countermeasure to non-weight-bearing soleus muscle atrophy. J. Appl. Physiol. 73: 1894-1899, 1992.

19. Kraemer, R. R., J. L. Kilgore, G. R. Kraemer, and V. D. Castracane. Growth hormone, IGF-I, and testosterone responses to resistive exercise. Med. Sci. Sports Exerc. 24: 1346$1352,1992$.

20. Lee, M. O., and N. K. Schaffer. Anterior pituitary growth hormone and the composition of growth. J. Nutr. 7: 337-363, 1933.

21. Linderman, J. K., K. L. Gosselink, R. E. Grindeland, F. W. Booth, and V. R. Mukku. The synergistic effect of exercise and growth hormone on skeletal muscle atrophy during hindlimb suspension (Abstract). FASEB J. 7: A3867, 1993.

22. Martin, T. P., V. R. Edgerton, and R. E. Grindeland. Influence of space flight on rat skeletal muscle. J. Appl. Physiol. 65: 2318-2325, 1988.

23. Nilsson, K. O., L. G. Heding, and B. Hoekfelt. The influence of short term submaximal work on plasma concentrations of catecholamines, pancreatic glucagon, and growth hormone in man. Acta Endocrinol. 69: 286-294, 1975.

24. Nwoye, L., W. F. H. M. Mommaerts, D. R. Simpson, K. Seraydarian, and M. Marusich. Evidence for a direct action of thyroid hormone in specifying muscle properties. Am. J. Physiol. 242 (Regulatory Integrative Comp. Physiol. 11): R401R408, 1982.

25. Roy, R. R., K. M. Baldwin, and V. R. Edgerton. The plasticity of skeletal muscle: effects of neuromuscular activity. In: Exercise and Sports Sciences Reviews, edited by J. Holloszy. Baltimore, MD: Williams \& Wilkins, 1991, vol. 19., p. 269-312.

26. Roy, R. R., R. E. Grindeland, E. Grossman, V. Mukku, R. Talmadge, and V. R. Edgerton. Growth hormone, insulinlike growth factor-1 and exercise interactions in maintaining muscle mass during hindlimb suspension of hypophysectomized rats (Abstract). ASGSB Bull. 6: 94, 1992.

27. Rudman, D. A., G. Feller, L. Cohn, K. R. Shetty, I. W. Rudman, and M. W. Draper. Effects of human growth hormone on body composition in elderly men. Horm. Res. Suppl. 36: 73-81, 1991.

28. Smith, P. K., R. I. Krohn, G. T. Hermanson, A. K. Mallia, F. H. Gartner, M. D. Provenzano, E. K. Fujimoto, N. M. Goeke, B. J. Olson, and D. C. Klenk. Measurement of protein using bicinchoninic acid. Anal. Biochem. 150: 76-85, 1985.

29. Thomason, D. B., and F. W. Booth. Atrophy of the soleus muscle by hindlimb unweighting. J. Appl. Physiol. 68: 1-12, 1990.

30. Thornton, W. E., and J. A. Rummel. Muscular deconditioning and its prevention in space flight. In: Proc. Skylab Life Sci. Symp., edited by R. S. Johnson and L. F. Dietlein. Houston, TX: NASA, 1974, p. 403-416.

31. Tischler, M. E., E. J. Henriksen, K. A. Munoz, C. S. Stump, C. R. Woodman, and C. R. Kirby. Spaceflight on STS-48 and earth-based unweighting produce similar effects on skeletal muscle of young rats. J. Appl. Physiol. 74: 2161-2165, 1993.

32. Tsika, R. W., R. E. Herrick, and K. M. Baldwin. Effect of anabolic steroids on skeletal muscle mass during hindlimb suspension. J. Appl. Physiol. 63: 2122-2127, 1987.

33. Tsika, R. W., R. E. Herrick, and K. M. Baldwin. Effect of anabolic steroids on overloaded and overloaded suspended skeletal muscle. J. Appl. Physiol. 63: 2128-2133, 1987.

34. Vasques, M., R. Grindeland, M. Martinelli, and R. Furlanetto. Effects of 7 days of microgravity on rat plasma hormone levels (Abstract). Physiologist 31: A104, 1988.

35. Wronski, T. J., and E. R. Morey-Holton. Skeletal responses to simulated weightlessness: a comparison of suspension techniques. Aviat. Space Environ. Med. 58: 63-68, 1987. 